Annales Geophysicae (2001) 19: 263-273 (C) European Geophysical Society 2001

\title{
Long term changes in EUV and X-ray emissions from the solar corona and chromosphere as measured by the response of the Earth's ionosphere during total solar eclipses from 1932 to 1999
}

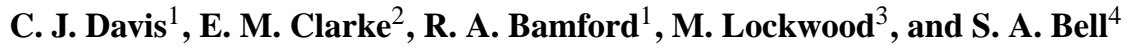 \\ ${ }^{1}$ Rutherford Appleton Laboratory, Chilton, Oxfordshire, UK \\ ${ }^{2}$ Sheffield Hallam University, Sheffield, Yorkshire, UK \\ ${ }^{3}$ Department of Physics and Astronomy, Southampton University, Southampton, UK \\ ${ }^{4} \mathrm{HM}$ Nautical Almanac Office, Rutherford Appleton Laboratory, Chilton, Oxfordshire, UK
}

Received: 2 August 2000 - Revised: 23 January 2001 - Accepted: 25 January 2001

\begin{abstract}
Measurements of the ionospheric E region during total solar eclipses in the period 1932-1999 have been used to investigate the fraction of Extreme Ultra Violet and soft $\mathrm{X}$-ray radiation, $\Phi$, that is emitted from the limb corona and chromosphere. The relative apparent sizes of the Moon and the Sun are different for each eclipse, and techniques are presented which correct the measurements and, therefore, allow direct comparisons between different eclipses. The results show that the fraction of ionising radiation emitted by the limb corona has a clear solar cycle variation and that the underlying trend shows this fraction has been increasing since 1932. Data from the SOHO spacecraft are used to study the effects of short-term variability and it is shown that the observed long-term rise in $\Phi$ has a negligible probability of being a chance occurrence.
\end{abstract}

Key words. Ionosphere (solar radiation and cosmic ray effects) - Solar physics, astrophysics, and astronomy (corona and transition region)

\section{Introduction}

From the very earliest days of ionospheric science, it was recognised that measurements of the Earth's ionosphere during total solar eclipses provided a unique opportunity to investigate the influence of the Sun on the Earth's upper atmosphere.

Initial measurements concentrated on the response time of the ionosphere in an attempt to determine whether the ionisation in the upper atmosphere was created by electromagnetic or corpuscular radiation (e.g. Mimno et al, 1933). Such experiments were technically very difficult at the time because radio sounding at many frequencies, required to locate the peak concentration of the ionisation, took an interval that

Correspondence to: C. J. Davis (c.j.davis@ rl.ac.uk) was long in comparison with the timescales of change of the ionosphere itself. Nevertheless, measurements were made with enough resolution to determine that the ionosphere was created by light rather than by particles.

Subsequent eclipse measurements were designed to investigate the chemical composition of the upper atmosphere and, in particular, to determine the recombination rate of the ionospheric plasma (e.g. Minnis, 1955). At lower ionospheric heights (the E layer), the amount of ionisation at a given time is governed by the production and loss processes (photochemical equilibrium). During an eclipse, it was reasoned, production of ionisation will cease and thus, the loss rate can be determined by studying the decay rate of the ionosphere. The results obtained from such experiments differed considerably from theoretical calculations and it was eventually realised that this difference was due to ionising radiation coming from the uneclipsed solar corona. Attempts to determine the loss rate by such measurements were abandoned in the mid 1960s when, with the advent of rocketry, in-situ measurements became possible.

Thereafter, the focus of eclipse measurements switched to the observation of atmospheric waves caused by the passage of the eclipse shadow travelling at supersonic speeds through the atmosphere. Waves are best detected at the focus of a curved eclipse path, away from the zone of totality. As a result, the more or less unbroken sequence of ionospheric measurements made under total eclipse conditions came to an end.

In 1999, interest in ionospheric measurements during eclipses was rekindled by investigations which revealed longterm changes in the strength of the magnetic field leaving the Sun and entering the heliosphere, the "coronal source flux" (Lockwood et al., 1999; Stamper et al., 1999). These changes correlate well with changes in the total solar irradiance (Lockwood and Stamper, 1999; Lean, 2000) and indicate that the percentage of radiation emitted by the solar 
corona should also have increased over the last century.

The solar radiation responsible for the production of ionisation in the $\mathrm{E}$ region ionosphere (at altitudes between about 100 and $140 \mathrm{~km}$ ) comes from two distinct regions of the electromagnetic spectrum, as soft X-rays and in the Extreme Ultra Violet (EUV). The optical depth of the atmosphere at these wavelengths is sufficiently large that the ionising radiation penetrates to the $E$ region without being significantly absorbed at higher altitudes. The behaviour of the $\mathrm{E}$ region during eclipses, therefore, provides an opportunity to investigate the fraction of EUV and soft X-ray radiation emitted by the corona. The E-region ionosphere (altitudes below about 140 $\mathrm{km}$ ) is produced by wavelength bands of about $1-20 \mathrm{~nm}$ and 80-102.7 nm. At wavelengths shorter than $1 \mathrm{~nm}$ and greater than $102.7 \mathrm{~nm}$ (the ionisation threshold for $\mathrm{O}_{2}$ molecules), radiation penetrates to the $\mathrm{D}$ region (below about $100 \mathrm{~km}$ ) and between 20 and $80 \mathrm{~nm}$, it primarily produces ionisation in the F-region (above about $140 \mathrm{~km}$ ). The variability of solar emissions at wavelengths $100 \mathrm{~nm}$ and greater has been studied on solar cycle and longer timescales (Lean, 2000), but little is known about long-term variations at the shorter wavelengths arising from the hotter regions of the chromosphere and corona. The fractional changes in spectral irradiance increase with decreasing wavelength, but the changes in absolute energy flux decrease (below a peak at around 300-400 $\mathrm{nm}$ ) because energy flux is much smaller at the shorter wavelengths. The strength of the emissions at less than $100 \mathrm{~nm}$ is likely to vary on solar cycle and 100-year timescales with changes in the coronal field, which is the source of the energy that heats the corona to temperatures of several million degrees. There are two factors that may influence the coronal heating: the magnitude of the field and the "form factor" that describes its complexity, and the number and strength of current sheets at which magnetic reconnection and heating can occur. The relationship of the coronal source flux to either is not yet known, and is also likely to vary with the sunspot cycle. On the one hand, a direct correspondence between the ionising flux from the corona and the coronal source flux is not expected. On the other hand, it is expected that the two will vary together in some way.

The total eclipse on 11 August 1999 was the first such event over the UK since 1927, and provided a timely opportunity to search for long-term variations in the corona. The resulting measurements of the ionospheric decay were used to estimate the percentage of ionising radiation emitted by the uneclipsed (limb) corona and chromosphere, and these have been compared with measurements made during an eclipse in July 1945 which occurred at a similar time of year, and at a similar phase of the solar cycle (Davis et al., 2000). This study indicated that the percentage of ionising radiation emitted by the corona had increased from $16 \%$ to $25 \%$ within this time. It was acknowledged in the study that this simple comparison did not take into account other possible causes of the increase, such as the relative angular size of the lunar disk in each case.

The purpose of the current study is to extend the initial comparison to include all available eclipse measurements spanning the period between 1932 and 1999, and to correct these for the relative angular size of the lunar disk in each case. We also use images from the SOHO spacecraft to study short-term variability of chromospheric and coronal emissions, and assess the implications for studies of longterm trends.

\section{Method}

The method used to calculate, $\Phi$, the percentage of the total ionising radiation incident on the E-region ionosphere at a given time during an eclipse, has been fully documented in a previous publication (Davis et al., 2000) and thus, only a brief description will be given here.

At E-region altitudes, where transport of ionisation is insignificant and the loss rate of ionisation is dominated by the dissociative recombination of $\mathrm{O}_{2}^{+}$ions, the rate of change of ionisation generated by solar radiation can be expressed as

$\frac{d N}{d t}=q_{0} C h(\chi)-\alpha N^{2}$

where $N$ is the number of electrons per cubic metre, $\alpha$ is the recombination rate, $q_{0}$ is the production rate for an overhead Sun, and $C h(\chi)$ is the Chapman function which describes the diurnal variation in $q_{0}$ with respect to the solar zenith angle, $\chi$.

During a solar eclipse, the production function is multiplied by $\Phi$, the fraction of ionising radiation that is not eclipsed at a given time. Comparing the eclipse day with a control day and assuming that $q_{0}$ is the same on the two days, $\Phi$ can be expressed as a function of $N$ and $t$

$\Phi=\frac{d N_{E} / d t+\alpha N_{E}^{2}}{d N_{C} / d t+\alpha N_{C}^{2}}$

where the subscripts $E$ and $C$ refer to the eclipse and control data, respectively.

The production function, $q$, is a product of the intensity, $I$, of the ionising radiation striking the atmosphere, the ionising efficiency, $\eta$, of the gasses in the atmosphere, the cross section for absorption of radiation, $\sigma$, and the neutral gas density, $n$. Although $q_{0}$ is assumed to be the same on the control and eclipse days, small variations can occur, due to the occurrence of transient phenomena, such as flares. In order to compensate for this, where possible, the control data is scaled to fit the trend in the eclipse data prior to the start of the eclipse. This would also compensate for any slight difference in neutral gas concentration or composition between the control and eclipse days. The value of $\Phi$ is not sensitive to the exact value of $\alpha$ and thus, it is sufficient to use a typical value and assign error bars that allow for the known uncertainty in $\alpha$. It is then possible to calculate $\Phi$ using the peak concentration of ionisation in the $\mathrm{E}$ layer, measured on the control and eclipse days, $N_{E}$ and $N_{C}$, from the corresponding E-layer critical frequencies, $(f o E)_{E}$ and $(f o E)_{C}$.

As discussed in the previous section, the solar radiation responsible for the production of ionisation in the $\mathrm{E}$ region 
comes from two distinct regions of the spectrum, namely, wavelengths of 1-20 nm (soft X-rays) and 80-102.7 nm (EUV). The behaviour of the $\mathrm{E}$ region during eclipses, therefore, provides an opportunity to investigate the fraction of radiation emitted by the limb corona, i.e. a form of average over these EUV and soft X-ray wavelength ranges.

\section{The total solar eclipses investigated in this study}

It has been possible to estimate values of $\Phi$ for all of the total eclipses since 1932 for which we have data. With the exception of the 1932 data, where the frequency was swept manually, measurements were automated and recorded on film (and in the case of later experiments to computer disk) where the relevant parameters could be carefully scaled after the event. The accuracy of such measurements is mainly dependent on the rate with which the ionograms were collected; the faster this rate, the more accurately the changes in the ionosphere can be estimated.

In the case of the 1932 data, the critical frequency was found by sounding at a selected frequency, using the time-offlight to determine whether the returned signal was reflected at $\mathrm{E}$ or $\mathrm{F}$ region altitudes, and then adjusting the frequency accordingly. In this way, it was possible to zero in on the E region critical frequency. During the eclipse, however, the ionosphere was changing faster than the operators could change frequency, resulting in a series of measurements containing few critical frequency values, and many measurements from the lower $\mathrm{F}$ region. It is still possible to use these data, however, as they represent an upper limit for $f_{o} E$, thus enabling an upper limit for $\Phi$ to be calculated. For the eclipse of 15 February 1961 (Nesterov, 1962), measurements were made from Nessebar, Bulgaria, where the eclipse occurred in the morning. The data presented in the literature does not start until just before the start of the eclipse, therefore, increasing the uncertainty when scaling the control data. It is also unclear from the text whether the control curve is derived from actual data, or simply an estimate based on typical behaviour. With these factors, it is probable that the estimate of $\Phi$ obtained from our analysis represents an overestimate. These factors are accounted for in our uncertainty estimates.

Two sets of data were identified for the eclipse of 20 May 1947, only one of which appears in the literature (Denisse and Kundu, 1947). The time resolution of this data is not high, with approximately 6 minutes between each $f o E$ value. Although totality lasted for 3 minutes and 29 seconds during this eclipse, this is still significantly less than the resolution of the data and thus, the value of $\Phi$ calculated from this data is also likely to be an overestimate. The second set of data from this eclipse was located in the archives of the World Data Center for Solar Terrestrial Physics, Boulder. This previously unpublished data sequence from Bocaiuva, Brazil has a much higher time resolution, increasing to a sounding every 15 seconds in the hours around totality. It is not surprising, therefore, that the value of $\Phi$ calculated from these data is lower than the value of $\Phi$ calculated from

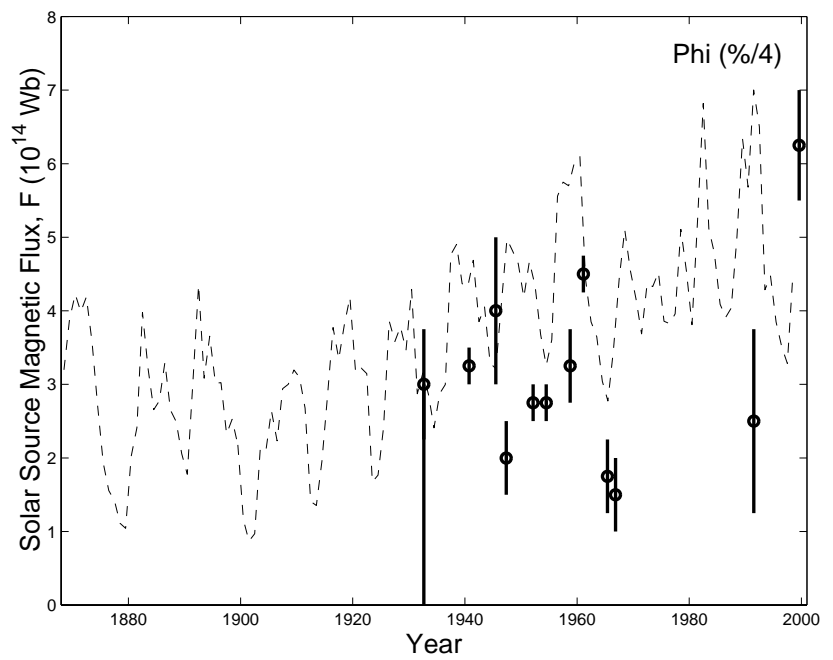

Fig. 1. Uncorrected $\Phi$ values (circles, with uncertainties given by the vertical bars) calculated from ionospheric measurements for 12 eclipses between 1932 and 1999. For comparison, the solar source magnetic flux (dashed line) estimated from the aa geomagnetic index is shown as a dashed line.

the previously published data (Denisse and Kundu, 1947). In the discussions and data plots that follow, therefore, the value of $\Phi$ quoted for 1947 will be taken from the Bocaiuva data.

Three other previously unpublished sequences of data are also used in this study: from Aitutaki in the Cook Islands during the eclipse of 20 May 1965; from Huancayo, Peru, during the eclipse of 12 November 1966; and from Maui, Hawaii, during the eclipse of 11 July 1991. All these have been scaled from films stored in the WDC archives in Boulder.

The eclipse of 11 July 1991 occurred early in the morning and as a result, there is not much data from which the control day can be estimated. As a result, it is possible that the value of $\Phi$ estimated from this data is slightly underestimated. In addition, this eclipse is the only one in our study which was not total at $\mathrm{E}$ region altitudes, reaching a maximum obscuration of $99.14 \%$. These factors are accounted for in the quoted uncertainty, and the data is included in this study to provide some comparison with the most recent eclipse data (from 1999).

Data from all the previously unpublished eclipses are presented in the appendix. Information for all the eclipses used in this study, including the relevant references (where appropriate) are presented in Table 1. Figure 1 presents the values of $\Phi$, derived for the 12 eclipses with all uncertainties combined into the error bars, estimated using the same method as applied by Davis et al. (2000). The dashed line gives the variation of the coronal source flux, as estimated from the $a a$ geomagnetic index, using the method of Lockwood et al. (1999): it reveals the solar cycle and long-term variations which we might expect to see mirrored in the eclipse data. However, as discussed in the introduction, we do not necessarily expect a direct correspondence. The values shown 


\begin{tabular}{|c|c|c|c|c|c|c|c|c|c|c|c|c|}
\hline 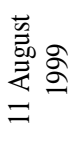 & 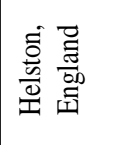 & 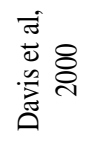 & 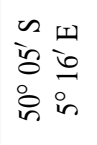 & 怃 & 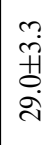 & 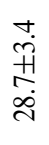 & $\underset{\widehat{\sigma}}{\stackrel{ }{\sigma}}$ & $\begin{array}{l}\qquad 0 \\
\infty \\
0 \\
0\end{array}$ & $\begin{array}{l}\stackrel{m}{i} \\
\stackrel{+}{H} \\
\stackrel{y}{+}\end{array}$ & 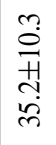 & $\stackrel{8}{0}$ & 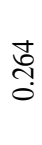 \\
\hline 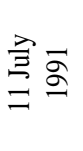 & 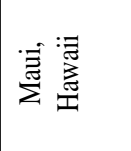 & & $\begin{array}{ll}z & 3 \\
\infty & 0 \\
o & 0 \\
0 & 0 \\
0 & 0 \\
0 & 0\end{array}$ & 哭 & $\begin{array}{l}0 \\
\dot{0} \\
\stackrel{+}{+}\end{array}$ & 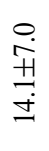 & & $\bar{\sigma}$ & 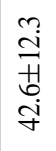 & 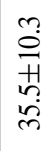 & 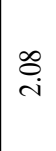 & $\frac{\circ}{0}$ \\
\hline 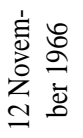 & 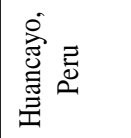 & & 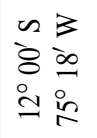 & तี & 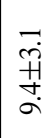 & 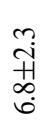 & $\stackrel{\infty}{\stackrel{\infty}{\sigma}}$ & $\begin{array}{l}\text { ڤ్ర } \\
\stackrel{0}{0}\end{array}$ & 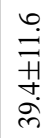 & $\begin{array}{l}\text { जे } \\
\text { iे } \\
\text { ले }\end{array}$ & $\vec{b}$ & $\stackrel{+}{8}$ \\
\hline 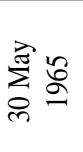 & 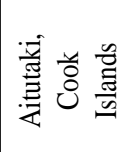 & & $\begin{array}{ll} & 3 \\
01 & 3 \\
i & 5 \\
0 & 0 \\
\infty & 0\end{array}$ & II & 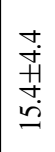 & 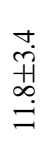 & & 守 & $\begin{array}{l}0 \\
\stackrel{0}{0} \\
+ \\
+ \\
+ \\
\dot{m}\end{array}$ & $\begin{array}{l}\infty \\
\infty \\
0 \\
+1 \\
\infty \\
\infty \\
\sim\end{array}$ & 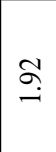 & స్ర \\
\hline 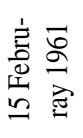 & 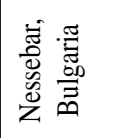 & 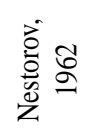 & 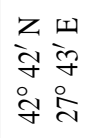 & $\begin{array}{l}\vec{H} \\
\infty\end{array}$ & 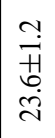 & $\frac{m}{\stackrel{m}{+H}}$ & 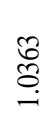 & 范 & 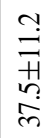 & $\begin{array}{l}\text { t. } \\
\text { Hे } \\
\text { ले }\end{array}$ & $\stackrel{0}{0}$ & ปี \\
\hline 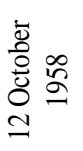 & 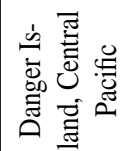 & 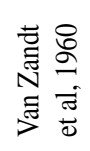 & $\begin{array}{l}n \\
\infty \\
\dot{\infty} \\
o \\
0 \\
0 \\
0\end{array}$ & İ & $\frac{\pi}{\stackrel{H}{a}}$ & 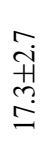 & $\underset{\hat{\sigma}}{\stackrel{i}{i}}$ & ڤ్రి & 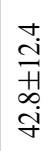 & $\begin{array}{c}m \\
\frac{m}{2} \\
+\frac{1}{2} \\
\dot{n} \\
m\end{array}$ & तె & $\frac{1}{0}$ \\
\hline 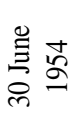 & 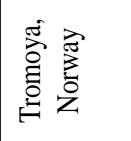 & 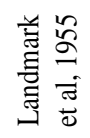 & $\begin{array}{ll}z & w \\
o & 1 \\
i & a\end{array}$ & $\ddot{H}$ & 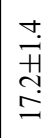 & $\frac{n}{\frac{n}{6}}$ & 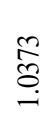 & $\bar{\Xi}$ & $\begin{array}{l}n \\
0 \\
0 \\
+1 \\
0 \\
\dot{m}\end{array}$ & $\begin{array}{l}\infty \\
\infty \\
0 \\
+ \\
+\infty \\
\infty \\
\dot{\sim}\end{array}$ & $\stackrel{q}{\stackrel{q}{-}}$ & $\stackrel{\vec{\circ}}{\circ}$ \\
\hline 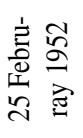 & 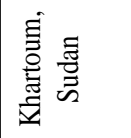 & 总令 & 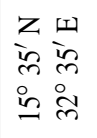 & 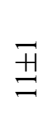 & 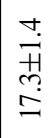 & 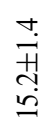 & 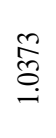 & $\stackrel{\overbrace{}}{\widehat{~}}$ & 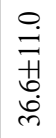 & 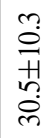 & $\stackrel{\circ}{\stackrel{\circ}{-}}$ & ج্ㅇㅇ \\
\hline 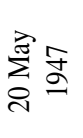 & 龍 & & $\begin{array}{ll} & 3 \\
\text { के } & 3 \\
0 & 0 \\
0 & 0 \\
1 & 9\end{array}$ & Î & $\frac{\pi}{\oplus}$ & $\begin{array}{l}0 \\
\text { iे } \\
\text { ? } \\
\stackrel{0}{0}\end{array}$ & 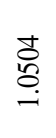 & $\frac{n}{a}$ & $\begin{array}{l}\stackrel{m}{i} \\
\stackrel{+}{+} \\
\stackrel{q}{q}\end{array}$ & $\begin{array}{l}m \\
\frac{a}{2} \\
\frac{H}{2} \\
\ddot{n}\end{array}$ & $\underset{i}{\stackrel{q}{i}}$ & : \\
\hline 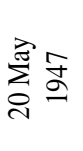 & 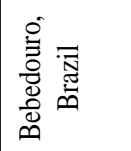 & 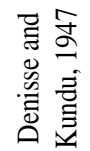 & 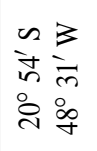 & $\stackrel{\oplus}{ \pm}$ & 贲 & 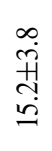 & 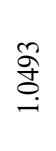 & $\frac{n}{a}$ & & & & \\
\hline 吝哭 & 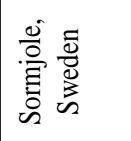 & 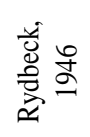 & 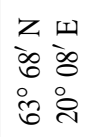 & $\underset{\text { 暮 }}{ }$ & 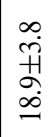 & $\begin{array}{l}0 \\
+ \\
+ \\
+ \\
\infty \\
\infty\end{array}$ & 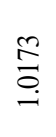 & $\stackrel{n}{\stackrel{0}{0}}$ & 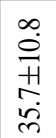 & $\begin{array}{l}\stackrel{0}{0} \\
+\vec{n} \\
\stackrel{i}{i}\end{array}$ & $\stackrel{\Xi}{I}$ & $\stackrel{\infty}{\stackrel{\circ}{0}}$ \\
\hline 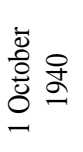 & 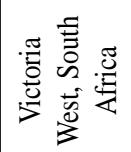 & 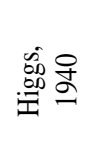 & 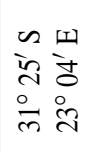 & $\underset{\Theta}{-\vec{H}}$ & 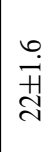 & $\begin{array}{l}\frac{n}{7} \\
\frac{H}{2}\end{array}$ & $\begin{array}{l}\stackrel{0}{\circ} \\
\stackrel{\leftrightarrow}{\circ} \\
\text {. }\end{array}$ & \begin{tabular}{l}
5 \\
\multirow{2}{0}{} \\
0
\end{tabular} & $\begin{array}{l}n \\
\stackrel{n}{\#} \\
\infty \\
\infty \\
\infty \\
\infty\end{array}$ & $\begin{array}{l}0 \\
0 \\
+1 \\
\text { i } \\
\text { m }\end{array}$ & 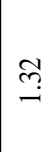 & $\stackrel{\sigma}{0}$ \\
\hline 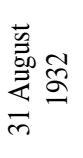 & 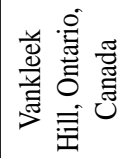 & 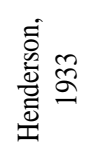 & 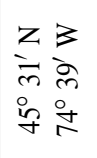 & $\stackrel{\text { H }}{\mathrm{I}}$ & 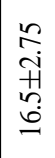 & 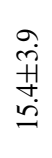 & ڤ̊ & ర్ర & \begin{tabular}{l}
0 \\
$\stackrel{0}{0}$ \\
+ \\
\multirow{H}{0}{} \\
$\dot{m}$
\end{tabular} & $\begin{array}{l}o \\
\infty \\
0 \\
o \\
o \\
o\end{array}$ & $\stackrel{\overbrace{}}{2}$ & 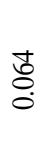 \\
\hline 芴 & 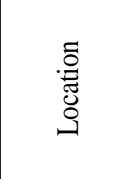 & 芯 & 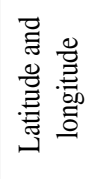 & $\theta$ & $\theta \theta$ & $\tilde{\theta}^{2}$ & $\begin{array}{l}\stackrel{5}{\hat{\Xi}} \\
+ \\
\vdots \\
=\end{array}$ & 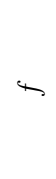 & $\begin{array}{l}b \\
H \\
Z\end{array}$ & $\mid \begin{array}{l}* \\
0 \\
H \\
{ }^{*} \\
3 \\
3\end{array}$ & 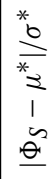 & $\hat{\theta}$ \\
\hline
\end{tabular}


were all taken at times of minimum $\Phi$ for each eclipse. Since the distribution of ionising radiation is not constant across the solar disk and corona, the minimum value of $\Phi$ may occur slightly before or after totality, depending on whether a particularly active region is exposed or occluded. However, particularly for the earliest eclipse measurements, the timing is not accurate enough to identify the exact time of mideclipse. For the most recent eclipse (Davis et al., 2000), the Global Positioning System (GPS) enabled very accurate timing (to well within a second). From these results, it can be seen that the minimum value of $\Phi$ is very similar to that at mid-totality and thus, it was considered more accurate to use the minimum $\Phi$ values for comparison in this analysis.

\section{Correcting for the apparent size of the lunar shadow}

Table 1 gives the ratio $(r+d r) / r$ for each eclipse, where $r$ is the angular radius subtended by the Sun, and $(r+d r)$ is the angular radius subtended by the Moon. We study here total eclipses, for which $d r>0$ and thus, $(r+d r) / r>1$. It can be seen that this ratio is different for every eclipse. Since a significant fraction of the radiation responsible for creating the Earth's ionosphere is emitted by the chromosphere and lower corona, it is likely that $\Phi$ is a sensitive function of the apparent diameters of the Sun and the Moon. It is, therefore, important to account for the effects of the changes in this ratio. Otherwise, the variation it will cause in $\Phi$ will mask other factors, such as solar cycle effects or long-term changes. Two procedures that attempt to account for the variation in the ratio of the apparent diameters are outlined below. In both cases, the value of $\Phi$ is corrected to represent the fraction of radiation that would have been incident on the ionosphere had the Sun and the Moon had the same apparent diameters $(d r=0)$. The difference between the two approaches concerns the assumptions made about the distribution of the ionising radiation as a function of distance from the edge of the solar disk.

\subsection{Method 1: A simple geometrical correction}

In the first method, the intensity of ionising radiation emitted by the lower corona is assumed to be the same as that emitted by the disk itself. Thus, a simple scaling factor can be applied to the values of $\Phi$ based on the area of the corona obscured by the lunar shadow.

The ratio of areas of the solar and lunar disks can be expressed as

$$
\frac{A_{L}}{A_{S}}=\frac{1-\Phi}{1-\Phi^{\prime}}
$$

where $A$ is the area, $\Phi$ is the percentage of uneclipsed ionising radiation flux, and $\Phi^{\prime}$ is the fraction of uneclipsed ionising radiation corrected to a Moon of the same apparent diameter as the Sun by assuming that the emission was constant over the area $A_{L}$. The subscripts $S$ and $L$ refer to the solar disk (of angular radius $r$ ) and the lunar disk (of angular ra- dius $r+d r$ ), respectively. Assuming circular lunar and solar disks, the ratio of the areas is

$$
\frac{A_{L}}{A_{S}}=\frac{\pi(r+d r)^{2}}{\pi r^{2}}=\left(\frac{r+d r}{r}\right)^{2}
$$

and so $\Phi^{\prime}$ can be written

$$
\Phi^{\prime}=1-(1-\Phi)\left(\frac{r}{r+d r}\right)^{2}
$$

Using this method, values of $\Phi^{\prime}$ at totality were calculated using the observed values of $\Phi$ and the ratio of the lunar and solar diameters, which can be computed for each eclipse with great accuracy. The results are quoted in Table 1 and plotted in Fig. 4 in comparison with the solar magnetic source flux calculated from the $a a$ geomagnetic index (dashed line), as in Fig. 1.

It can be seen that the correction of $\Phi$ values in this way increases the value for every eclipse used in this study $\left(\Phi^{\prime}>\right.$ $\Phi)$. This occurs because no annular eclipses were used $(d r<$ $0)$ and thus, the ratio $(r+d r) / r$ is always greater than unity. The degree to which each $\Phi$ value is corrected is dependent on the magnitude of this ratio. The most dramatic effect of this correction is to enhance the solar cycle variation that can be seen in the $\Phi^{\prime}$ values (shown in Fig. 4), in comparison with the original $\Phi$ values (shown in Fig. 1). This occurs because, by chance, the solar minimum eclipses happened to have relatively large values of the ratio $(r+d r) / r$.

This correction technique has the advantage that it is simple to apply. However, it is by no means comprehensive, ignoring as it does, any variation of emission as a function of distance from the solar disk and any changes in the structure of the corona throughout the solar cycle. In order to investigate these and attempt to account for them, a second correction method was devised.

\subsection{Method 2: Using solar data}

Inspection of any EUV image of the Sun will demonstrate that the emission of ionising radiation is not uniform around the limb (or indeed, across the disk itself). Furthermore, the form of the corona seen in eclipses or by a coronograph varies considerably during the solar cycle. The distribution of this radiation, at any one wavelength, will depend on the location of active regions, the number, position and intensity of which vary according to the phase of the solar cycle. Although the effects of this radiation could be more accurately accounted for using information from satellites such as SOHO (Domingo et al., 1995), this information is not available for all but the most recent eclipses.

Data from the Extreme-ultraviolet Imaging Telescope (EIT) onboard the SOHO satellite were used to simulate the effects of the 11 August 1999 eclipse. One of the wavelengths measured by this instrument, $17.1 \mathrm{~nm}$, falls within one of the two bands of the solar spectrum responsible for ionising the Earth's atmosphere. Although this is not a comprehensive measure of all the wavelengths responsible for creating the ionospheric E-region, it does assist in identifying 


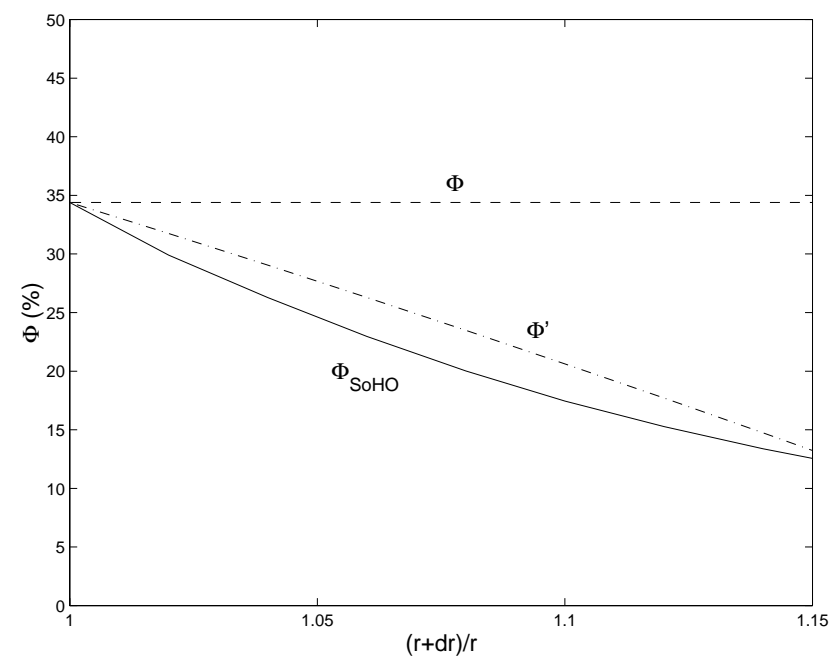

Fig. 2. A comparison of three functions describing the variation of the unobscurred ionising radiation, $\Phi$, with the ratio of lunar and solar radii, $(r+d r) / r$. Here the dash-dotted line (labelled $\left.\Phi^{\prime}\right)$ represents $\Phi$ values adjusted using a simple geometric correction (as given by Eq. 5). It can be seen that this closely matches the solid line labelled $\Phi_{\mathrm{SOHO}}$, which represents the observed variation, as calculated from data from the EIT instrument on SOHO taken during the eclipse of 11 August 1999. The uncorrected $\Phi$ value is also plotted for comparison.

the distribution of the ionising radiation across the solar disk and into the lower corona. A full-Sun image from 11 August 1999 at this wavelength was used to simulate a series of artificial eclipses by blocking off radiation from a central disk with a range of diameters. For each diameter ratio, the intensity of all unobscured pixels was integrated and presented as a fraction, $\Phi_{\mathrm{SOHO}}$, of the integration over the whole image. The results are presented in Fig. 2 (solid line), compared with values of $\Phi$ and $\Phi^{\prime}$ (dashed and dot-dashed lines, respectively) calculated for the same range of diameter ratios using correction factors of unity and as given by Eq. (5), respectively. This figure clearly shows that correcting the $\Phi$ values to give values of $\Phi^{\prime}$ using Eq. (5) more closely represents the variation of $\Phi_{\mathrm{SOHO}}$ calculated from actual solar data for the day of the eclipse (i.e. $\left|\Phi^{\prime}-\Phi_{\mathrm{SOHO}}\right|<\left|\Phi-\Phi_{\mathrm{SOHO}}\right|$ ). Although using values of $\Phi_{\mathrm{SOHO}}$ to correct for the size of the lunar disk would be more accurate than the simple $\Phi^{\prime}$ correction for the 1999 eclipse, such solar images are not available for any of the other eclipses for which we have ionospheric data and thus, similar curves cannot be generated.

In order to investigate the solar cycle variation of the distribution of ionising radiation, further simulations with EIT data were carried out using six months of data from around solar minimum and six months nearer solar maximum. One sample image per day was analysed from between July and November 1996 and from between September 1999 and March 2000. Curves for these six-month averages of $\Phi_{\text {SOHO }}$ are presented as solid lines in Fig. 3. The dashed lines represent the curves at plus and minus one standard

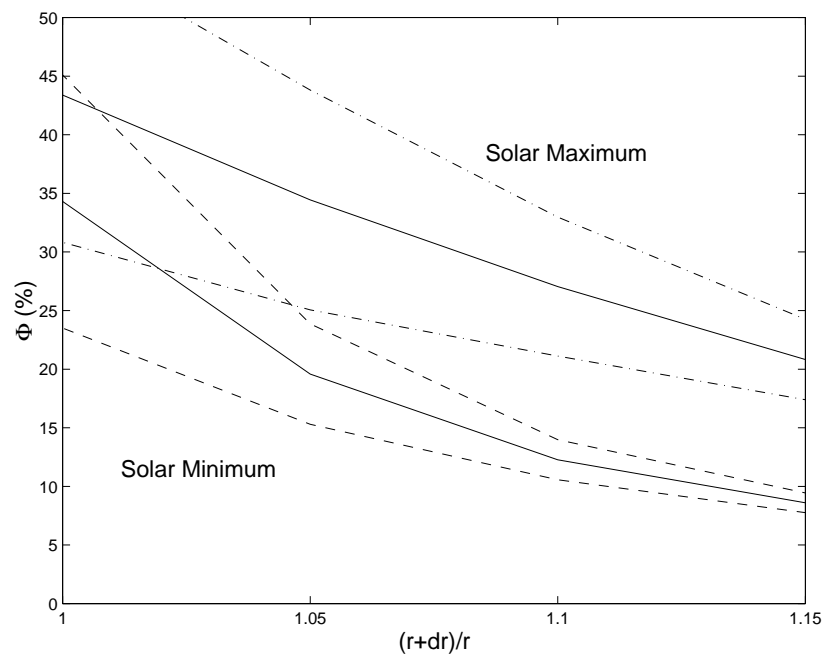

Fig. 3. The mean variation of $\Phi_{\mathrm{SOHO}}$ with $(r+d r) / r$ for 2 six month periods at solar minimum and solar maximum (solid lines). The variations of plus and minus one standard deviation from these mean values is represented by the dash-dot and dashed lines (for sunspot maximum and sunspot minimum, respectively).

deviation around the sunspot minimum curve, with the dot dashed lines representing the same for the sunspot maximum period. The results show that the correction to $\Phi$ to allow for $(r+d r) / r$ will vary between solar maximum and minimum. As previously discussed for the August 1999 event, the values of $\Phi_{\mathrm{SOHO}}$ at solar maximum closely match the simple correction method which produced values of $\Phi^{\prime}$, indicating that the intensity of radiation in the lower corona is reasonably constant at solar maximum. At solar minimum, however, the $\Phi$ values fall off more rapidly as a function of $(r+d r) / r$. This quantitatively shows how the distribution of ionising radiation emitted by the solar corona varies with solar cycle. Note that for the whole corona $(d r=0)$, the relative brightness depends on the solar cycle, as one would expect, giving $\Phi_{\mathrm{SOHO}}$ values of $43.5 \pm 12.5$ at sunspot maximum and $34.0 \pm 10.5$ at sunspot minimum.

In order to account for the variation between solar maximum and solar minimum conditions, the phase of the solar cycle was characterised for each eclipse by calculating the sunspot number on that date, as a fraction $f$ of the range of sunspot numbers in that cycle. The corrected $\Phi$ value appropriate to that phase of the solar cycle was then calculated using a weighted sum of the value obtained from the solar maximum correction, $\Phi_{\text {smax }}$, with the value obtained from the solar minimum correction, $\Phi_{\text {smin }}$. Before this could be done, however, the two curves were normalised so that they gave a value of 1 for $(r+d r) / r=1$ (this is because the curves are only needed to correct from the actual value of $(r+d r) / r$ to a standard value of unity; they are not used to obtain absolute values of $\left.\Phi_{\mathrm{SOHO}}\right)$. If the solar cycle phase estimate were $f$, then the final correction would be

$\Phi_{S}=f \Phi_{\mathrm{smin}}+(1-f) \Phi_{\mathrm{smax}}$. 


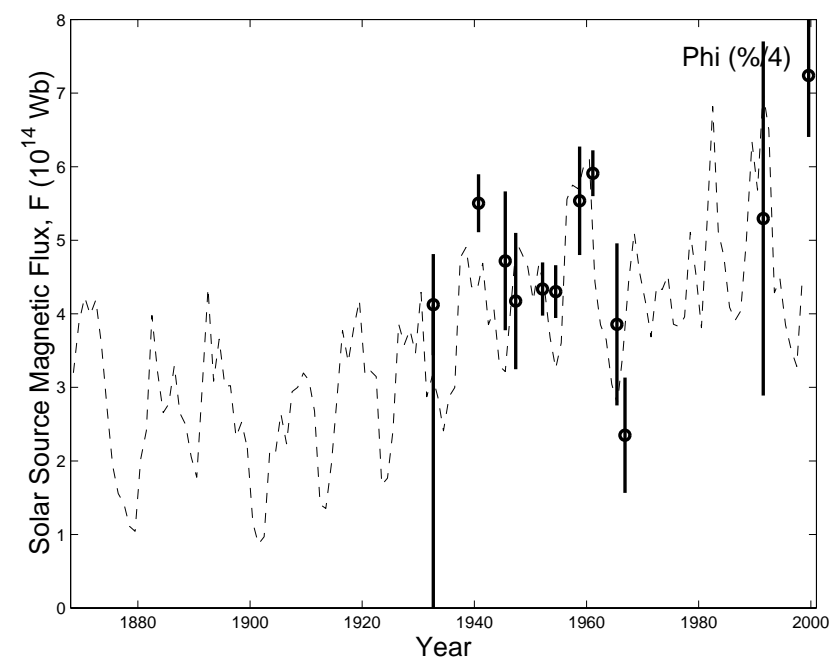

Fig. 4. The same as for Fig. 1 for $\Phi^{\prime}$, the $\Phi$ values that have been corrected to account for the variation of $(r+d r) / r$ for each eclipse using the simple geometric formula given in Eq. 5 .

The results of this correction are presented in Fig. 5. As for the $\Phi^{\prime}$ estimates, all $\Phi_{S}$ values calculated using Eq. (6) are higher than the original $\Phi$ values (since $d r>0$ for all the eclipses used). It can be seen that there are some significant differences, most notably, in the value calculated from the 1991 eclipse over Maui, for which the $\Phi_{S}$ value (i.e. the value from the ionospheric data, corrected using a cycle-dependent limb correction derived from the SOHO data) is considerably smaller than the equivalent value of $\Phi^{\prime}$ (the ionospheric data corrected using the simpler Eq. 5).

It should be noted that neither of these methods for correcting the observed values of $\Phi$ is likely to be definitive, since the first assumes that the solar limb is of uniform brightness, while the second represents some average solar cycle behaviour at one specific wavelength. In contrast, the ionospheric response for each eclipse corresponds to a specific interval in solar activity integrated over all ionising wavelengths. While this is the case, it is apparent that it is necessary to account for the variation in eclipse conditions and that a simple correction is considerably better than not correcting the data at all. Comparison of Figs. 1, 4 and 5 shows that either correction method introduces a solar cycle variation (thus, this has not simply arisen from the cycle-dependent correction used in Fig. 5). In all three cases, the largest value of the obscuration factor $\left(\Phi, \Phi_{S}\right.$ or $\left.\Phi^{\prime}\right)$ is for the 1999 eclipse, and the corrected values $\left(\Phi_{S}\right.$ and $\left.\Phi^{\prime}\right)$ show variations that have similarities in terms of both solar cycle and longer-term changes, with the coronal source flux variation (dashed line).

\section{Discussion and conclusions}

Although the two methods discussed above produced different correction factors for the values of $\Phi$, there are some

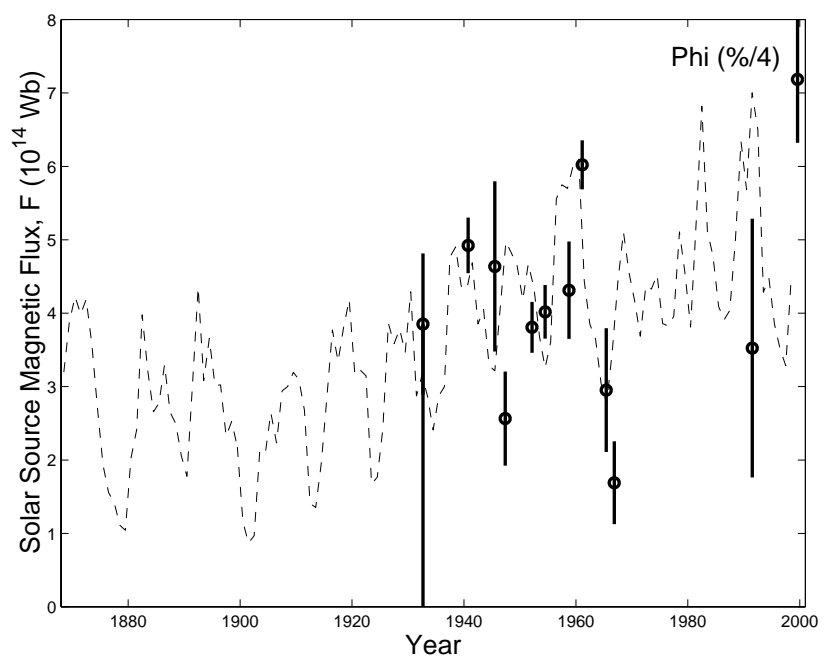

Fig. 5. The same as for Fig. 1 for $\Phi_{S}$, the $\Phi$ values that have been corrected to account for the variation of $(r+d r) / r$ using the curves presented in Fig. 3. The degree to which each of these two curves is used in the correction factor is determined by the estimate $f$ of the phase of the solar cycle during that eclipse, based on the sunspot number.

common elements that emerge from both techniques. Both the $\Phi^{\prime}$ and $\Phi_{S}$ sequences contain a solar cycle variation that is not so apparent in the raw $\Phi$ values due to the variety of the $(r+d r) / r$ ratios. In the case of $\Phi_{S}$, the correction method itself could have introduced a solar cycle variation. However, a solar cycle variation is also seen in $\Phi^{\prime}$, for which the correction does not depend on the phase of the solar cycle. It is apparent that the inherent assumption in the correction used to generate $\Phi^{\prime}$, concerning the distribution of ionising radiation across the solar limb, more closely resembles solar maximum conditions, as shown by the simulation using the data from the EIT instrument on SOHO (compare Figs. 2 and 3 ). This would result in the correction at solar minimum being smaller than it ought to be, and if this were the case, values of $\Phi^{\prime}$ at solar minimum have been underestimated. Another common feature in both $\Phi^{\prime}$ and $\Phi_{S}$ estimates is the large values for the 1999 eclipse, in comparison with all of the previous measurements. This is despite the fact that the EIT data from SOHO shows that the coronal emissions at $17.1 \mathrm{~nm}$ were lower than average at the time of this particular eclipse. We note that ionospheric observations of the 1999 event were also made from Chilton, UK, where the photosphere was only eclipsed by $97 \%$ at E-region altitudes: analysis of these data supports the high value of $\Phi$ deduced from the Helston instrument. This lends credibility to this one data point, despite the absence of any eclipse data from the years immediately preceding this eclipse.

The eclipse measurements are "snapshots" of the condition of the corona, and we need to consider the longer-term changes in $\Phi_{S}$ and $\Phi^{\prime}$, as shown in Figs. 4 and 5, in the context of the effects of short-term variability of the corona. For the 1999 event, we estimate that the solar cycle phase factor 


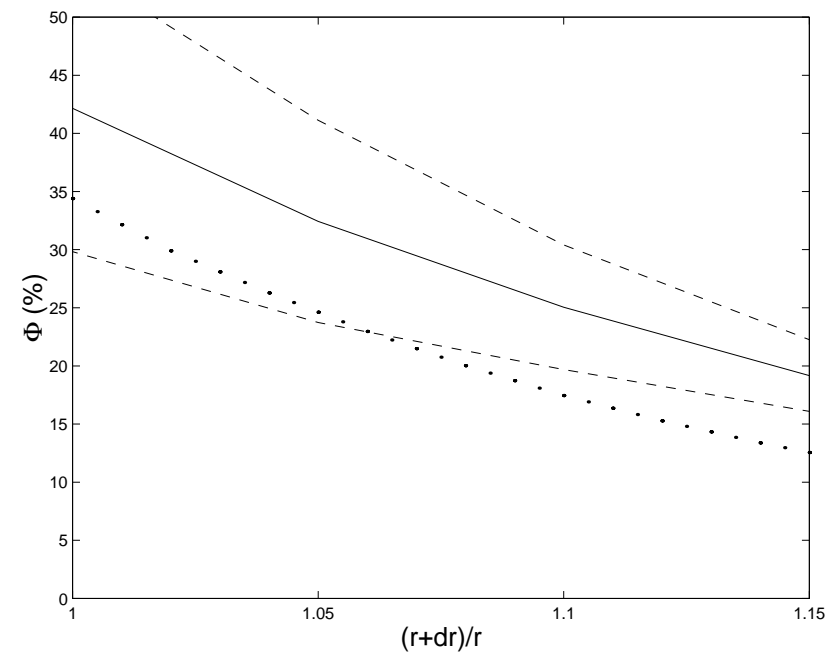

Fig. 6. The variation of $\Phi_{\mathrm{SOHO}}$ with $(r+d r) / r$, calculated from SOHO EIT data taken during the eclipse of 11 August 1999 (dotted line) in comparison with the mean value $\mu$ for 50 days' data at the same phase of the solar cycle (interpolated from the two mean curves presented in Fig. 3). At all $(r+d r) / r$, the corrected value $\Phi_{S}$, estimated for the ionospheric eclipse data of 11 August 1999, is lower than the mean value $\mu$ for that phase of the cycle. The dashed lines are at plus and minus one standard deviation $\sigma$ from the mean $\mu$.

$f$ is 0.865 . Using this value, the average variation of $\Phi_{\mathrm{SOHO}}$ with $(r+d r) / r$ has been interpolated from the solar maximum and minimum curves, as shown in Fig. 3: the result $\mu$ is given by the solid line in Fig. 6 . The dashed lines give the curves that are plus and minus one standard deviation $\sigma$ from this mean $\mu$. For comparison, the dotted line in Fig. 6 gives the variation of $\Phi_{\text {SOHO }}$ that is taken from the EIT image at the time of the eclipse on 11 August 1999. This comparison shows that at the time of this eclipse, the corona was less bright than the average (for that phase of the solar cycle), such that $\Phi_{\text {SOHO }}$ was lower by about $8 \%$ at all $(r+d r) / r$ values. The difference was less than one standard deviation at $(r+d r) / r$ near unity, but was more than one standard deviation at $(r+d r) / r$ above approximately 1.06. This shows that it was the corona beyond 1.06 solar radii, in particular, that was less bright than average. If we take $d r=0$, we find that at the time of the eclipse, $\Phi_{\mathrm{SOHO}}=34 \%$, whereas the mean value, for that phase of the solar cycle, is $\mu=42.5 \%$ (with a standard deviation $\sigma$ of $12.3 \%$ ). Thus, the value during the eclipse is $\left(\Phi_{\mathrm{SOHO}}-\mu\right) / \sigma=0.63$ standard deviations from the mean. For the observed Gaussian distribution, we can deduce that the probability of deviating this far from the mean was 0.26. Thus, we can conclude from the EIT data during the 11 August 1999 eclipse that, in terms of its $\Phi_{\mathrm{SOHO}}$ value, the corona was less bright than average for that phase of the solar cycle, but that it was not exceptionally different.

However, just as the SOHO data show us that short-term variability resulted, by chance, in a lower-than-average value being obtained on 11 August 1999, it is possible that the lower values obtained from the ionospheric experiments during the previous eclipses were also just a chance occurrence. Therefore, we need to assess the probabilities of this being the case.

The best corrected value from the ionospheric experiment on 11 August 1999 is $\Phi_{S}=28.7 \pm 3.4 \%$, whereas, as discussed above, the EIT image at $17.1 \mathrm{~nm}$ gives $\Phi_{\mathrm{SOHO}}=$ $34 \%$. Such a difference is to be expected because the ionospheric value represents an average over the soft X-ray (1$20 \mathrm{~nm}$ ) and EUV (80-102.7nm) wavelength bands, whereas $\Phi_{\mathrm{SOHO}}$ is the value for $17.1 \mathrm{~nm}$. If we assume that the spectrum retains the same shape, we can apply the same correction factor $\Phi_{S} / \Phi_{\text {SOHO }}=0.844$ to all events. Thus for each event, we computed the expected means and standard deviations, $\mu$ and $\sigma$, for the relevant phase of the current solar cycle (using $f$ ), as calculated above for the 11 August 1999 event. These were then multiplied by the factor of 0.844 to give $\mu^{*}$ and $\sigma^{*}$, the predicted mean and standard deviation for the ionospheric estimate. We then estimated the $\Phi_{S}$ value in terms of the number of standard deviations, i.e. we evaluated $\left(\Phi_{S}-\mu^{*}\right) / \sigma^{*}$. From this, we computed $P_{\Phi S}$, the probability of deviating from $\mu^{*}$ by at least this amount. The lowest 5 rows of Table 1 give the values of $f, \mu, \sigma, \mu^{*}, \sigma^{*}$, $\left(\left|\Phi_{S}-\mu^{*}\right| / \sigma^{*}\right)$ and $P_{\Phi S}$ for each event.

The values of $P_{\Phi S}$ are significant because they give us the probability that the $\Phi_{S}$ estimate derived was lower than the average (for the corresponding phase of the current solar cycle), by chance, arising from the short-term variability. Table 1 shows that the $\Phi_{S}$ estimates, particularly the earlier ones, are consistently low and that the probability of this being explained by short-term variability is also low, with all $P_{\Phi S}$ values before 1961 falling below 0.1 .

From Table 1 we can consider the "null hypothesis" that there is no long-term change in the $\Phi_{S}$ values and that the consistently lower values before 1967 , as compared with the recent solar cycle (monitored by SOHO and seen during the 1999 eclipse event), were chance occurrences and caused by short-term coronal variability. Since all events are independent of each other, the probability of this is the multiplicative product of all the $P_{\Phi S}$ probabilities, which equals $4 \times 10^{-16}$ and thus is negligible.

Therefore, we conclude that the 1999 eclipse did give significantly higher $\Phi$ values than any of the previous experiments, showing that a larger fraction of the relevant soft Xray and EUV wavelengths came from the limb corona. The data do suggest a solar cycle variation superimposed on a long-term drift. However, the paucity of data in the period between 1966 and 1999 makes it impossible to draw any definitive conclusions concerning the form of the increasing trend in the emission of ionising radiation from the solar corona. It is, therefore, important to add to the most recent observation by making similar measurements during eclipses, whenever possible. 


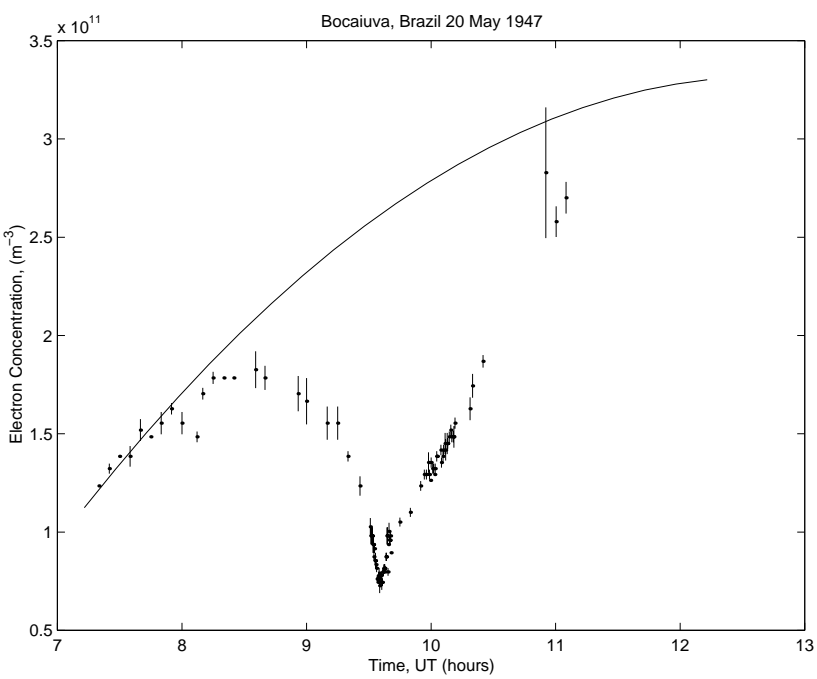

Fig. A1. The variation of the E region peak electron concentration with time during the eclipse of 20 May 1947 at Bocaiuva, Brazil (points). The control day is represented by a polynomial fit to the data from an adjacent day (solid line).

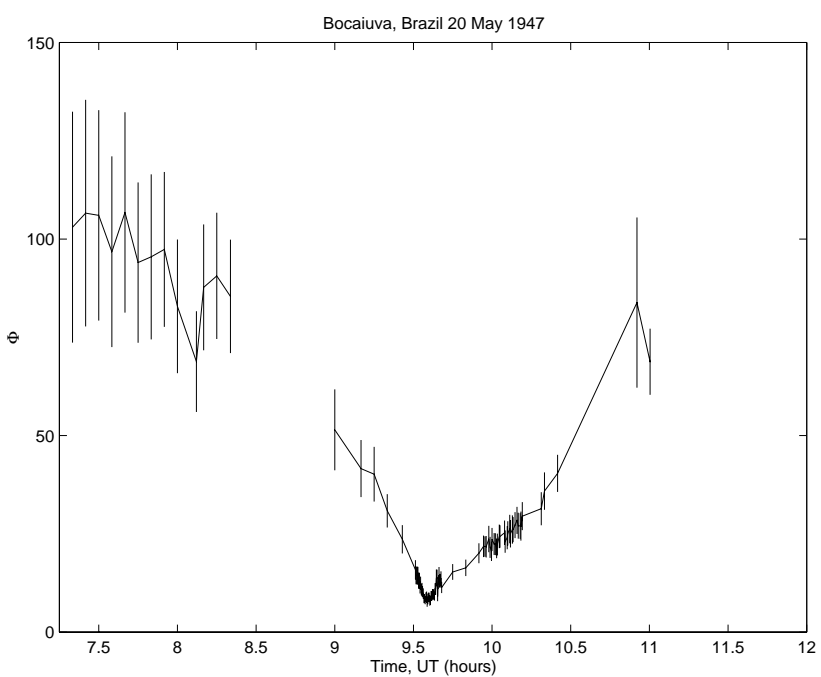

Fig. A2. The variation of $\Phi$ during the eclipse of 20 May 1947 at Bocaiuva, Brazil as calculated using Eq. (2) and the data shown in Fig. A1.

\section{Appendix A}

For most of the eclipses listed in Table 1, the raw data on the peak E-layer concentrations during the eclipse and on the control days ( $N_{E}$ and $N_{C}$, respectively), or alternatively the corresponding critical frequencies $(f o E)_{E}$ and $(f o E)_{C}$, are presented in the cited publications. However, in four of the cases, the data have not previously appeared in the literature. These are the eclipses that took place on: 20 May 1947 (observed from Bocaiuva, Brazil); 30 May 1965 (Aitutaki, Cook Islands); 12 November 1966 (Huancayo, Peru); and 11 July 1991 (Maui, Hawaii). In this appendix, we present the data for these four cases for completeness. In each case, we

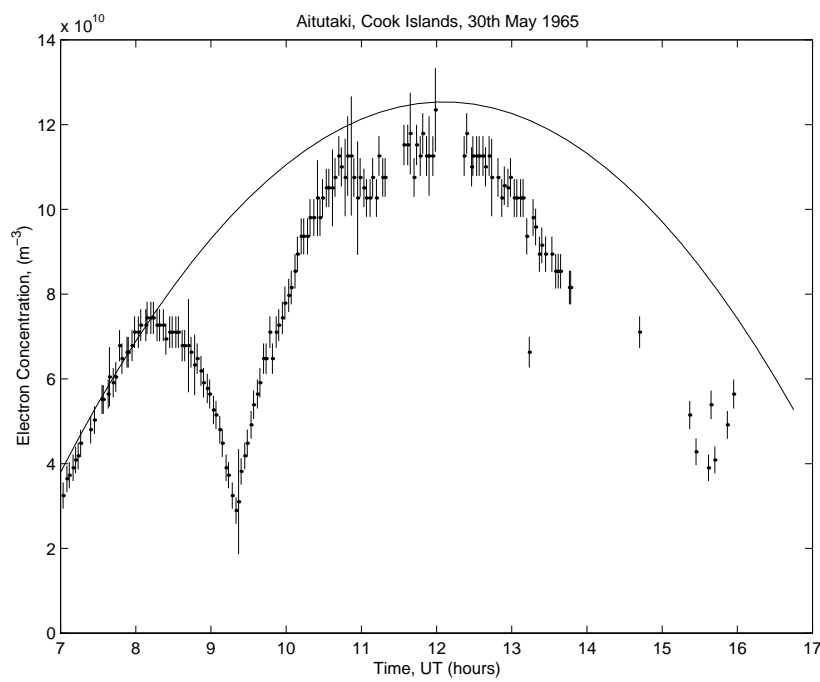

Fig. A3. The variation of the E region peak electron concentration with time during the eclipse of 30 May 1965 at Aitutaki, Cook Islands (points). The control day is represented by a polynomial fit to the data from an adjacent day (solid line).

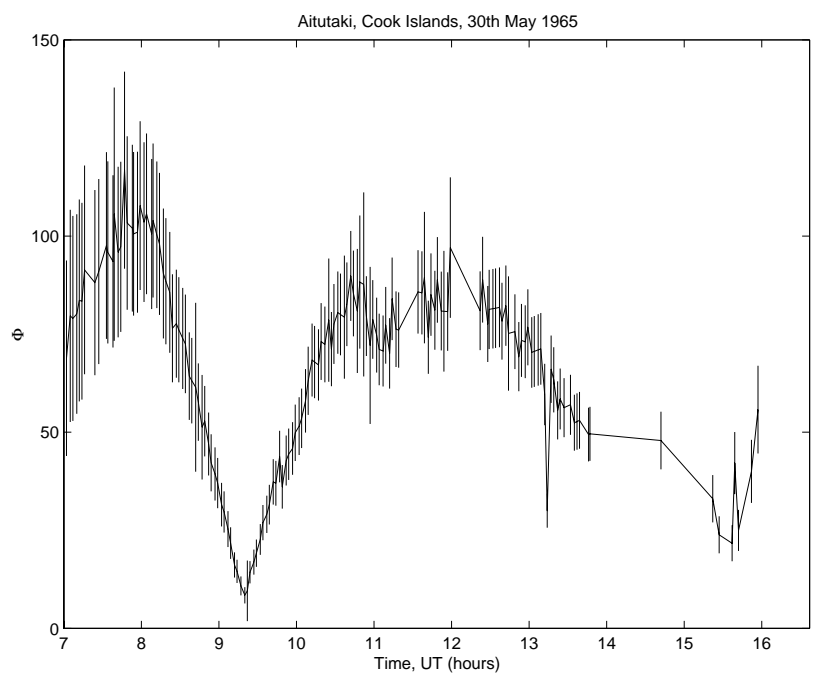

Fig. A4. The variation of $\Phi$ during the eclipse of 30 May 1965 at Aitutaki, Cook Islands, as calculated using Eq. (2) and the data shown in Fig. A3.

present the observed values of $N_{E}$ as data points, along with a solid line that is a polynomial fit to the control day data $N_{C}$. We also present the corresponding values of $\Phi$ on a separate plot, with error bars estimated using the method given by Davies et al. (2000).

The data for Bocaiuva (20 May 1947) do not extend much beyond the time of the eclipse (Fig. A1). Thus, the control day is based on the form of the variation on adjacent days, scaled to fit the data taken before first contact. Thus, the corresponding $\Phi$ values (Fig. A2) are near $100 \%$, but the data do not cover the return to this value. Note, as for all events, that error bars are smallest near the minimum of $\Phi$, where the value given in Table 1 is scaled. The sample rate of 


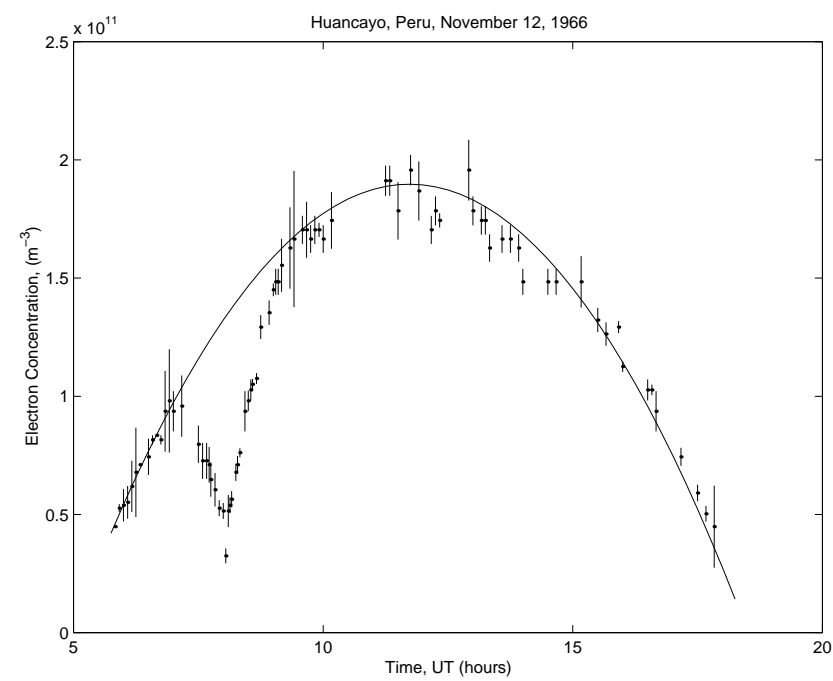

Fig. A5. The variation of the E region peak electron concentration with time during the eclipse of 12 November 1966 at Huancayo, Peru (points). The control day is represented by a polynomial fit to the data from an adjacent day (solid line).

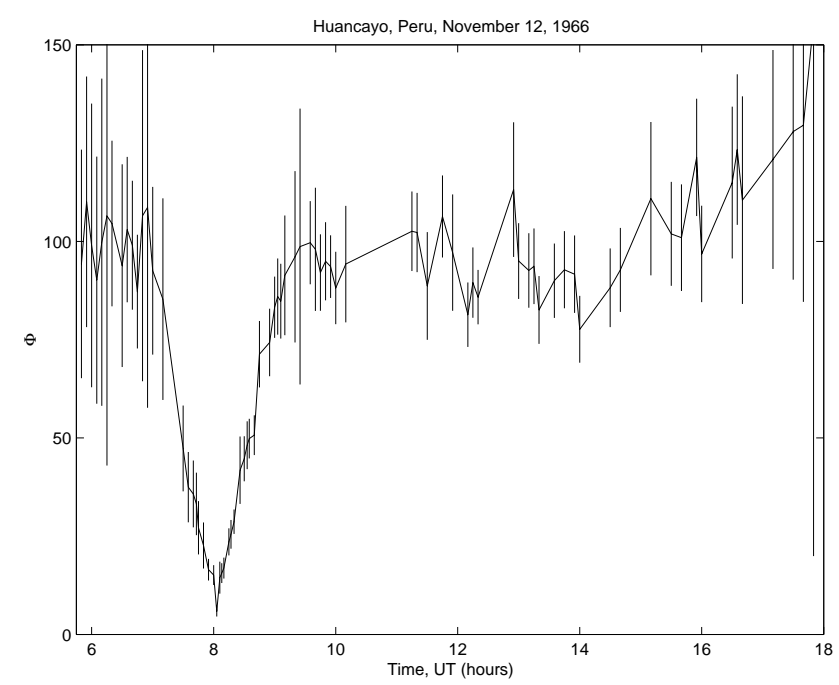

Fig. A6. The variation of $\Phi$ during the eclipse of 12 November 1966 at Huancayo, Peru as calculated using Eq. (2) and the data shown in Fig. A5.

the data near minimum is very high for these measurements, and thus, the minimum in $\Phi$ is very well defined.

For the Aitutaki event (30 May 1965), the overall form of the control day does not match well the variation seen away from the eclipse (Fig. A3). This suggests that the upper atmospheric conditions were particularly affected at this site. This is reflected in the $\Phi$ values (Fig. A4) which never return to $100 \%$ after the event.

On the other hand, the data outside the event seen at Huancayo on 12 November 1966, do match the control data exceptionally well (Fig. A5) so that $\Phi$ values (Fig. A6) are around $100 \%$, both before and after the event.

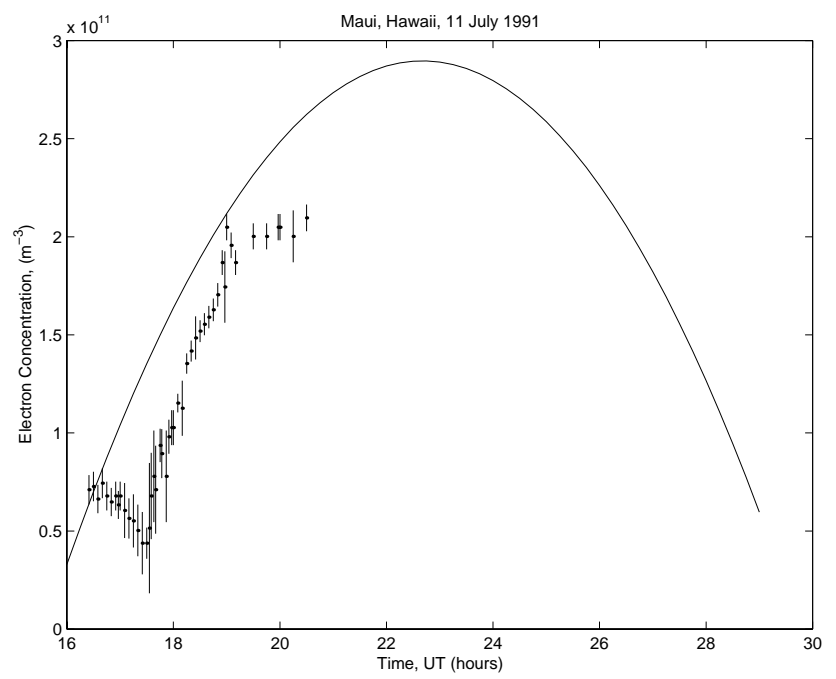

Fig. A7. The variation of the E region peak electron concentration with time during the eclipse of 11 July 1991 at Maui, Hawaii (points). The control day is represented by a polynomial fit to the data from an adjacent day (solid line).

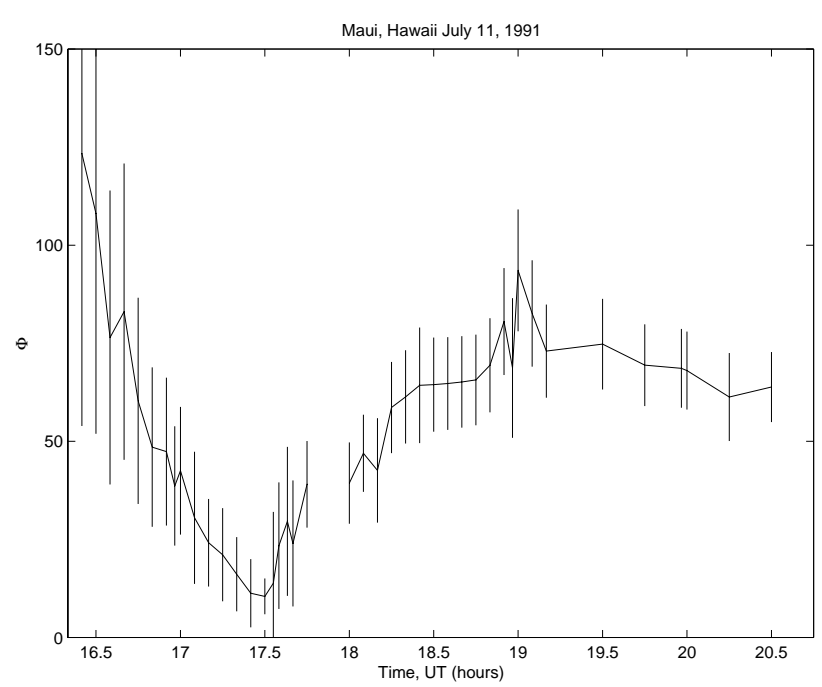

Fig. A8. The variation of $\Phi$ during the eclipse of 11 July 1991 at Maui, Hawaii as calculated using Eq. (2) using the data shown in Fig. A7.

For the event seen at Maui on 11 July 1991, data are only available for around the time of the eclipse (Fig. A7). Nevertheless, there are indications that the thermosphere has changed significantly as $\Phi$ values (Fig. A8) only briefly return to $100 \%$. The lack of data from before the event (because it was close to sunrise) makes the control day variation less certain than for the other events. This uncertainty causes the larger error bar for the corresponding data point in Figs. 1, 4 and 5. Due to the lack of pre-event data, it is possible that the control day densities Nc are too high, in which case, the minimum $\Phi$ would be an underestimate. A value close to the upper limit of the uncertainty would make the $\Phi$ value 
for this event more similar to the 11 August 1999 event.

Acknowledgements. The authors wish to thank the World Data Centres for Solar Terrestrial Physics at Chilton and Boulder for the supply of ionospheric data and in particular to J. W. Wright and R. Conkright for their help, advice and for providing the facilities to scale previously unpublished data. They are also grateful to the SOHO EIT team for providing data from the EIT instrument. SOHO is a collaboration between ESA and NASA. This paper is dedicated to all those involved in collecting the ionospheric data used in this study.

Topical Editor E. Antonucci thanks J. Lilensten for his help in evaluating this paper.

\section{References}

Davis, C. J., Lockwood, M., Smith, J. A., Bell, S. A., and Clarke, E. M., Ionospheric measurements of relative coronal brightness during the total solar eclipses of 11 August 1999 and 9 July 1945, Ann. Geophysicae, 18, 182-190, 2000.

Denisse, J. F. and Kundu, M. R., Ionosperiques effectuees pendant l'eclips totale du soliel du 20 Mai 1947, C. R. Acad. Sci. Paris, 225, 1169-1171, 1947.

Domingo, V., Fleck, B., and Poland, A. I., The SOHO mission - an overview, Solar Phys., 162, 1-37, 1995.

Henderson, J. T., Measurements of ionisation in the kennellyheaviside layer during the solar eclipse of 1932, Can. J. Phys., 8, 1-14, 1933.

Higgs, A. J., Ionospheric measurements made during the total solar eclipse of 1 October 1940, Mon. Not. R. A. S., 102, 24-34, 1942.

Landmark, B., Lied, F., Orhaug, T., and Skribeland, S., Ionospheric observations during the solar eclipse of 30 June 1954, Norwegian Defence Research Establishment Report No. 8, December 1955.

Lean, J., Evolution of the Sun's spectral irradiance since the Maunder minimum, Geophys. Res. Lett., in press, 2000.

Lockwood, M. and Stamper, R., Long-term drift of the coronal source magnetic flux and the total solar irradiance, Geophys. Res. Lett., 26, 2461-2464, 1999.

Lockwood, M., Stamper, R., and Wild, M. N., A doubling of the Sun's coronal magnetic field during this century, Nature, 399, 6735, 437-439, 1999.

Mimno, H. R. and Wang, P. H., Continuous Kennelley-Heaviside layer records of a solar eclipse (with suggestions of a corpuscular effect on the Appleton layer). Proc. Inst. Radio Engrs., 21, 529545, 1933.

Minnis, C. M., Ionospheric behaviour at Khartoum during the eclipse of 25 February 1952, J. Atmos. Terr. Phys., 6, 91-112, 1955.

Nestorov, G. and Taubenheim, J., Untersuchungen an der ionosphärischen E-Schicht während der totalen Sonnenfinsternis 15 Februar 1961, J. Atmos. Terr. Phys., 24, 633-642, 1962.

Rydbeck, O. E. H., Chalmers solar eclipse ionospheric expedition 1945, Chalm. Tekn. Högsk. Handl., 53, 1946.

Stamper, R., Lockwood, M., Wild, M. N., and Clark, T. D. G., Solar causes of the long-term increase in geomagnetic activity, J. Geophys. Res., 104, 28325-28342, 1999.

Van Zandt, T. E., Norton, R. B., Stonehocker, G. H., Photochemical rates in the equatorial $\mathrm{F}_{2}$ region from the 1958 eclipse, J. Geophys. Res., 65, 5, 2003-2009, 1960. 